\title{
Prognostic Indices for Advance Care Planning in Primary Care: A Scoping Review
}

\author{
Peter Kim, MD, MPH, Jeanette M. Daly, PhD, RN, Maresi A. Berry-Stoelzle, PhD, MD, \\ Megan E. Schmidt, MEd, MPH, LeAnn C. Michaels, BS, David A. Dorr, MD, MS, and \\ Barcey T. Levy, PhD, MD
}

Background: Patient identification is an important step for advance care planning (ACP) discussions. Objectives: We conducted a scoping review to identify prognostic indices potentially useful for initiating ACP.

Methods: We included studies that developed and/or validated a multivariable prognostic index for all-cause mortality between 6 months and 5 years in community-dwelling adults. PubMed was searched in October 2018 for articles meeting our search criteria. If a systematic review was identified from the search, we checked for additional eligible articles in its references. We abstracted data on population studied, discrimination, calibration, where to find the index, and variables included. Each index was further assessed for clinical usability.

Results: We identified 18 articles with a total of 17 unique prognostic indices after screening 9154 titles. The majority of indices $(88 \%)$ had c-statistics greater than or equal to 0.70 . Only 1 index was externally validated. Ten indices, 8 developed in the United States and 2 in the United Kingdom, were considered clinically usable.

Conclusion: Of the 17 unique prognostic indices, 10 may be useful for implementation in the primary care setting to identify patients who may benefit from ACP discussions. An index classified as "clinically usable" may not be easy to use because of a large number of variables that are not routinely collected and the need to program the index into the electronic medical record. ( $\mathrm{J}$ Am Board Fam Med 2020;33:322-338.)

Keywords: Advance Care Planning, Electronic Health Records, Independent Living, Primary Health Care, Prognosis

In the United States and worldwide, there is recognition that advance care planning (ACP) is important in patient care. ${ }^{1-3}$ A group of experts using the Delphi process defined ACP as follows: "ACP is a process that supports adults at any age or stage of

This article was externally peer reviewed.

Submitted 8 May 2019; revised 11 October 2019; accepted 14 October 2019.

From the Department of Family Medicine, University of Iowa Carver College of Medicine, Iowa City, IA (PK, JMD, MAB-S, MES, BTL); Department of Epidemiology, University of Iowa College of Public Health, Iowa City, IA (BTL); Oregon Rural Practice-based Research Network, Oregon Health \& Science University, Portland, OR (LCM); Department of Medical Informatics and Clinical Epidemiology, Oregon Health and Science University, Portland, OR (DAD).

Funding: This work was partially supported by the Iowa Academy of Family Physicians Endowed Chair for Rural Medicine Fund, Department of Family Medicine, University of Iowa Carver College of Medicine, and the PatientCentered Outcomes Research Institute (PCORI) Award health in understanding and sharing their personal values, life goals, and preferences regarding future medical care. The goal of ACP is to help ensure that people receive medical care that is consistent with their values, goals and preferences during serious and chronic illness." allows physicians and other health care professionals

(PLC-1609-36277). The funders had no role in any part of the study including its conception and design, acquisition, or analysis and interpretation of data, and drafting or revision of the manuscript. The statements in this publication are solely the responsibility of the authors and do not necessarily represent the views of the PCORI, its board of governors, or methodology committee.

Conflict of interests: None.

Corresponding author: Barcey T. Levy, MD, PhD, Professor and Iowa Academy of Family Physicians Endowed Chair of Rural Medicine, Family Medicine, Department of Family Medicine, Carver College of Medicine, University of Iowa, 200 Hawkins Drive, Iowa City, IA 52242 (E-mail: barcey-levy@uiowa.edu). 
to provide care concordant with patient-defined goals and values. ${ }^{5}$ ACP is not limited to ensuring the designation of a proxy for health care decisionmaking or documentation of code status, although these aspects are usually part of the discussion.

Although ACP can reduce anxiety and depression in patients and families and increase the likelihood for patients to receive medical care concordant with their goals and values, ${ }^{6-8}$ only about one-third of the population in the United States participates in some form of ACP. ${ }^{9}$ Current research efforts focus on expanding the implementation of ACP and measuring its quality and clinical impact. ${ }^{10,11}$

Although ACP is potentially appropriate for nearly all adult patients, given the realities of a busy practice, it would be useful to have a system for identifying patients with a more limited prognosis. Family medicine physicians are well situated to engage in ACP due to the continuity of care that they provide. ${ }^{12}$ However, they are often uncertain about which patients to involve in ACP conversations and when to have the discussion. ${ }^{13}$ Prognosis is often used for referral to hospice or palliative care. ${ }^{14-16}$ Prognosis is a possible trigger for primary care physicians to initiate ACP with patients. ${ }^{17}$

We conducted a scoping review and summarized prognostic indices that predict all-cause mortality in community-dwelling adults. The purpose was to identify prognostic indices potentially useful for supporting implementation of ACP in primary care. The key question was the following: "What studies developed and/or validated a prognostic index for 6-month to 5-year all-cause mortality in community-dwelling adults?" Our objective was to identify indices that might assist family physicians and others with identifying patients who may be appropriate for ACP discussions well before the final weeks of life.

\section{Methods}

This review was informed by the Preferred Reporting Items for Systematic Reviews and Meta-analyses Extension for Scoping Reviews checklist. ${ }^{18}$

\section{Eligibility Criteria}

We adapted the following criteria from the Transparent Reporting of a multivariable prediction model for Individual Prognosis Or Diagnosis (TRIPOD) checklist $^{19}$ : the study developed and/or
Figure 1. Final Search Query as displayed on PubMed.

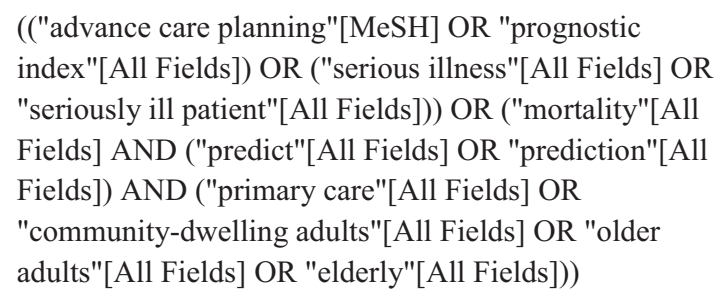

validated a multivariable prediction model in community-dwelling adults, and the outcome was allcause mortality in the range of 6 months to 5 years. The time frame was chosen to aid clinicians in initiating ACP before a patient is eligible for hospice but when a serious illness conversation would be appropriate. ${ }^{8,14}$ Individuals with a life expectancy of less than 6 months ideally should undergo a series of ACP conversations, but the focus of this article was to identify algorithms that could help predict life expectancy for longer intervals and, thus, promote ACP earlier than it might otherwise be done. All types of articles meeting our eligibility criteria were included.

\section{Information Sources and Search Strategy}

PubMed was searched on October 27, 2018 for articles using the strategy indicated in Figure 1. PubMed includes all articles from 1966 or the first year a given journal was published. Search terms were built using concepts such as ACP, prognostic index, serious illness, and mortality. Filters such as 'humans," "English-language," and "adult: 19+ years" were applied. References of a systematic review that met the eligibility criteria were checked to identify additional studies.

\section{Selection of Sources of Evidence}

The literature search and screening of titles were completed by 1 author (PK). Articles with titles indicating development and/or validation of a prognostic index in community-dwelling adults were chosen. Then, the abstracts of these articles were reviewed by 2 independent investigators (PK and JD/BTL/MBS) for possible inclusion. Differences were resolved by discussion among all reviewers. Full-text articles of abstracts that met our inclusion criteria were assessed for eligibility by 1 researcher (PK). During full-text review, studies were excluded if (1) a prognostic index was not internally 
validated, (2) it identified individual predictors of mortality but did not develop a usable index, or (3) over half of the cohort used to develop the index was hospitalized or nursing home patients. Eighteen articles met the inclusion criteria. ${ }^{20-37}$

\section{Data Abstraction}

Two investigators (PK, BTL) reviewed each article and tabulated each prognostic index according to broad categories of usability (clinically usable vs not usable) and summarized key information regarding each index in Tables 1 to 3 . Final tables were agreed on by all authors. An index was considered clinically usable if the instrument scoring and interpretation were available either in the article or online and not usable otherwise. A website link to each index, if available, was included in Table 3.

Discrimination of a prognostic index, as measured by the c-statistic in the cut-point analyses of the index, was categorized as poor $(<0.60)$, moderate ( 0.60 to 0.69 ), good ( 0.70 to 0.79 ), very good ( 0.80 to $0.89)$, or excellent $(\geq 0.90)$. $^{38,39}$ Tools were considered well calibrated if the percent difference between predicted and observed mortality in a given risk group was less than 10 and poor if greater than or equal to $10 \% .^{38}$ Other calibration and fitting methods, such as Hosmer-Lemeshow statistics, a test where statistical significance indicates poor calibration, and Cox calibration regression, where an $\alpha$ intercept of 0 and $\beta$ slope of 1 indicate perfect calibration, were included if reported. ${ }^{40}$ If the index predicted mortality at more than 1 time point, it was categorized under the longest mortality estimate that did not exceed 5 years, but information regarding the authors' other cut points was included in Table 2.

\section{Results}

\section{Search Results}

Figure 2 shows the flow diagram of the study selection process, adapted from the Preferred Reporting Items for Systematic Reviews and Meta-analyses Extension statement. ${ }^{41}$ Using our predefined search terms, we identified 18,305 records; applying filters on PubMed excluded 9,151 records and 9,154 titles were screened. After the title screening process, 182 abstracts were reviewed for potential eligibility. Forty-four full-text articles were assessed, and 1 additional unique article was found in the references of a systematic review identified through the search, ${ }^{38}$ leading to 45 articles that were considered for full-
Table 1. Descriptive Statistics of the 17 Unique Indices*

\begin{tabular}{|c|c|c|}
\hline & $\mathrm{n}$ & $\%$ \\
\hline \multicolumn{3}{|l|}{ Time Frame for Mortality } \\
\hline 6 month $^{20,21}$ & 1 & 5.9 \\
\hline 1 year $^{22-26}$ & 5 & 29.4 \\
\hline 15 month $^{27}$ & 1 & 5.9 \\
\hline 2 year $^{28}$ & 1 & 5.9 \\
\hline 3 year $^{29,30}$ & 2 & 11.7 \\
\hline 4 year $^{31}$ & 1 & 5.9 \\
\hline 5 year $^{32-37}$ & 6 & 35.3 \\
\hline \multicolumn{3}{|l|}{ Country } \\
\hline United States $^{20,21,25,26,28,30,31,34-37}$ & 10 & 58.8 \\
\hline United Kingdom ${ }^{22,23,33}$ & 3 & 17.6 \\
\hline Italy $^{24,27}$ & 2 & 11.8 \\
\hline Russia $^{29}$ & 1 & 5.9 \\
\hline South Korea ${ }^{32}$ & 1 & 5.9 \\
\hline \multicolumn{3}{|l|}{ C-statistics } \\
\hline 0.50 to 0.59 (poor) & 0 & 0 \\
\hline 0.60 to 0.69 (moderate) $^{29,30}$ & 2 & 11.7 \\
\hline 0.70 to 0.79 (good) $^{22,23,25,31-34,38}$ & 8 & 47.1 \\
\hline 0.80 to 0.89 (very good) $^{20,21,24,26-28,36,37}$ & 7 & 41.2 \\
\hline 0.90 to 1.00 (excellent) & 0 & 0 \\
\hline \multicolumn{3}{|l|}{ Calibration } \\
\hline Well calibrated & 13 & 76.5 \\
\hline$<10 \%$ Difference $22,24,26-28,30,31,34,35,37$ & 10 & 58.8 \\
\hline Hosmer-Lemeshow $P>.05^{33,36}$ & 2 & 11.8 \\
\hline $\begin{array}{l}\text { Cox calibration regression }{ }^{25} \text { (perfect } \\
\text { calibration: } \alpha=0, \beta=1 \text { ) }\end{array}$ & 1 & 5.9 \\
\hline Poorly calibrated (>10\% difference) $)^{32}$ & 1 & 5.9 \\
\hline Calibration curve only ${ }^{20,21}$ & 1 & 5.9 \\
\hline Not reported ${ }^{23,29}$ & 2 & 11.8 \\
\hline \multicolumn{3}{|l|}{ Usability $^{\dagger}$} \\
\hline Clinically usable $22,26-28,30,31,33,34,36,37$ & 10 & 58.8 \\
\hline Not usable $20,21,23-25,29,32,35$ & 7 & 41.2 \\
\hline
\end{tabular}

*Han et al. ${ }^{20}$ and Duarte et al. ${ }^{21}$ use the same index.

${ }^{\dagger}$ Usability: usable if the mortality risk can be calculated using the instrument and interpreted without referring to the text of the article and not usable otherwise.

text review. Of these, 18 articles met our inclusion criteria and were summarized in Tables 1 to $3 .^{20-37}$ Of these, 1 study externally validated a published index, ${ }^{21}$ yielding a total of 17 unique indices. Table 1 summarizes the 17 indices. The majority of indices were developed in the United States $(n=10)$, followed by Europe $(n=6)$, and Asia $(n=1)$.

The systematic review conducted by Yourman et al. ${ }^{38}$ identified 16 unique prognostic indices that were developed in community, nursing home, and hospital settings. Five out of 6 prognostic indices in the community setting for predicting 1 - to 5 -year mortality were captured using the search terms in this scoping review. ${ }^{27,28,30,31,37}$ The combined 
comorbidity score to predict 1-year mortality by Gagne et al. ${ }^{26}$ was not captured, possibly because it is listed under the medical subjects heading term "hospital mortality" on PubMed. It met our inclusion criteria and was included in the final list for full-text review. The remaining 10 of 16 studies in Yourman et al. ${ }^{38}$ were excluded based on our eligibility criteria.

\section{Characteristics of Identified Indices}

Table 2 summarizes the 18 articles meeting the criteria for full review, including the population studied, information on the development and/or validation cohort, calibration statistics, and discrimination as assessed by the c-statistic. Calibration and discrimination should be described for clinical prediction models. ${ }^{42}$ There was heterogeneity in reporting the calibration of prognostic indices, but the majority of indices were well calibrated, as indicated by less than $10 \%$ difference in the predicted and observed mortality rates. ${ }^{22,24,26-28,30,31,34,35,37}$ Two studies did not report calibration. ${ }^{2,29}$ No prognostic indices had excellent discrimination (cstatistic, $\geq 0.90$ ). Eight indices had very good discrimination (c-statistic, 0.80 to 0.89 ), 22,23,25,31-35 7 had good discrimination (c-statistic, 0.70 to $0.79),{ }^{21,24,26-28,36,37}$ and 2 had moderate discrimination (c-statistic, 0.60 to 0.69$).{ }^{29,30}$

Table 3 groups the indices by clinical usability. For each index, we report authors and year published, population and country, mortality time frame, where to find, and all variables included in the instrument. Ten articles presented their prognostic indices either in the article or online and were classified as clinically usable. $22,26-28,30,31,33,34,36,37$ There was $100 \%$ agreement between the 2 reviewers (PK and BTL) on clinical usability. Although Pilotto et al. ${ }^{24}$ included a link to downloadable software, we classified it as not usable because it requires knowledge of Italian. The modified Geriatric Prognostic Index by Jung et al..$^{32}$ is available as a free downloadable application on mobile devices, but we classified it as not usable because it requires knowledge of Korean and uses scales not commonly used in the United States.

\section{Assessment of Individual Indices}

Summarized below are 17 published indices identified from 18 articles according to the time frame of the mortality index.

\section{Six-Month Mortality}

Duarte et al. ${ }^{21}$ externally validated the PatientReported Outcome Mortality Prediction Tool (PROMPT) in patients age 65 and older in Maine, US. The development cohort was that used by Han et al. ${ }^{20}$ The PROMPT questionnaire shown in their Appendix is a patient self-reported questionnaire that takes 15 minutes. The calibration curve was shown, but no information was provided on predicted or observed mortality rates for the different risk groups. The index had good discrimination.

Han et al. ${ }^{20}$ developed PROMPT, which estimates 6-month mortality risk by using cohorts from the 1998 to 2003 Medicare Health Outcomes Survey of community-dwelling adults aged 65 years and older in the United States. The calibration curve was shown, but no actual data were provided for the predicted or observed mortality rates for the different risk groups. The index had good discrimination.

\section{One-Year Mortality}

The QMortality ${ }^{\circledR}$ risk prediction equation developed by Hippisley-Cox and Coupland is a 1-year mortality index for primary care patients aged 65 and older. $^{22}$ Using a large, validated medical research database in England, the algorithms for both men and women were well calibrated and had very good discrimination.

Crooks et al. ${ }^{23}$ developed a comorbidity score to predict 1 -year mortality using 3 national administrative databases in England. All people older than 20 years registered to a primary care practice were followed for 1 year and were randomly divided into 2 halves for development and validation. Characteristics of each cohort were not reported separately. The relative goodness of fit was statistically significant compared with the Charlson and the Elixhauser indices (likelihood ratio test, $P<.0001$ ), indicating improvement in model fitting for the score developed by Crooks et al. ${ }^{23}$ It also had better discriminatory performance than Charlson ${ }^{43}$ (c-statistic, 0.87 ; 95\% CI, $0.87-0.87$ ) or Elixhauser comorbidity measures ${ }^{44}$ (c-statistic, 0.87; 95\% CI, 0.87-0.87). Charlson ${ }^{43}$ and Elixhauser ${ }^{44}$ are wellknown prognostic indices.

Pilotto et al. ${ }^{24}$ developed an index based on an assessment of community-dwelling adults older than 65 years living at home in Italy. The index was 
Table 2. Prognostic Indices for Community-Dwelling Adults, by the Most Recent Published Year and Authors in Alphabetical Order, according to the Increasing Order of the Time Frame of Mortality Index

\begin{tabular}{|c|c|c|c|c|c|c|c|c|c|c|c|}
\hline \multirow[b]{5}{*}{ Author } & \multirow[b]{5}{*}{ Index } & \multirow{3}{*}{\multicolumn{2}{|c|}{ Population }} & \multicolumn{8}{|c|}{ Accuracy* } \\
\hline & & & & \multirow{2}{*}{\multicolumn{5}{|c|}{ Development }} & \multirow{2}{*}{\multicolumn{3}{|c|}{ Validation }} \\
\hline & & & & & & & & & & & \\
\hline & & \multirow[b]{2}{*}{ Development } & \multirow[b]{2}{*}{ Validation } & \multirow{2}{*}{\multicolumn{5}{|c|}{ Development $\uparrow$}} & \multirow{2}{*}{\multicolumn{2}{|c|}{ Validation }} & \\
\hline & & & & & & & & & & & \\
\hline \multicolumn{12}{|c|}{ 6-month all-cause mortality } \\
\hline \multirow[t]{2}{*}{$\begin{array}{l}\text { Duarte et al. } \\
(2015)^{21}\end{array}$} & \multirow{2}{*}{$\begin{array}{l}6 \text {-mo in } \\
\text { inpatient and } \\
\text { outpatient age } \\
\geq 65 \mathrm{y}\end{array}$} & \multirow[t]{2}{*}{$\begin{array}{l}\text { See Han et al. } \\
(2012)^{20}\end{array}$} & \multirow{2}{*}{$\begin{array}{l}\mathrm{n}=467 \\
\text { Mean age } 80 \mathrm{y} \\
56 \% \text { female } \\
7 \% \text { 6-mo mortality }\end{array}$} & \multicolumn{8}{|c|}{$\begin{array}{l}\text { Graph only (Figure } 2 \text { of paper) })^{21} \text {; used Hosmer-Lemeshow statistic, } p=0.66 \text {, indicates the } \\
\text { updated version of the original model is well calibrated }\end{array}$} \\
\hline & & & & $\begin{array}{l}C=0.75 ; \text { sa } \\
(2012)\end{array}$ & coho & used & y Han et al. & $C=0.73(0$ & -0.82 & & \\
\hline $\begin{array}{l}\text { Han et al. } \\
(2012)^{20}\end{array}$ & $\begin{array}{l}\text { 6-mo in } \\
\text { Medicare }\end{array}$ & $\begin{array}{l}\mathrm{n}=21,870 \\
\text { Mean age } 78 \text { y }\end{array}$ & $\begin{array}{l}\text { Used } 10 \text {-fold cross } \\
\text { validation of }\end{array}$ & Graph only & gure & of pal & & & & & \\
\hline & $\begin{array}{l}\text { beneficiaries } \\
\text { age } \geq 65 \mathrm{y}\end{array}$ & $\begin{array}{l}59 \% \text { female } \\
15 \% \text {-mo mortality }\end{array}$ & development cohort & $C=0.75$ & & & & $C$-statistic $\mathrm{N}$ & & & \\
\hline 1-year all-caus & mortality & & & & & & & & & & \\
\hline Hippisley-Cox & 1-y primary & $\mathrm{n}=1,466,598$ & $\mathrm{n}=499,478$ & NR & & & & Women & & & \\
\hline $\begin{array}{l}\text { \& Coupland } \\
(2017)^{22}\end{array}$ & $\begin{array}{l}\text { care patients } \\
\text { age } \geq 65 \mathrm{y}\end{array}$ & $\begin{array}{l}\text { Mean age } 75 \text { y } \\
45 \% \text { male }\end{array}$ & $\begin{array}{l}\text { Mean age } 75 \text { y } \\
45 \% \text { male }\end{array}$ & & & & & $\begin{array}{l}\text { Risk } \\
\text { percentile }\end{array}$ & $\begin{array}{l}\text { Pred } \\
1-y\end{array}$ & & $\begin{array}{l}\text { Observed, } \\
1-\mathrm{y}(\%)\end{array}$ \\
\hline & & $95 \%$ white or not & $95 \%$ white or not & & & & & $\geq 50^{\text {th }}$ & 13.5 & & 13.1 \\
\hline & & recorded & recorded & & & & & $\geq 90^{\text {th }}$ & 36.1 & & 35.4 \\
\hline & & $12 \% 1-y$ mortality & $12 \% 1-y$ mortality & & & & & $\geq 98^{\text {th }}$ & 59.4 & & 50.6 \\
\hline & & & & & & & & Men & & & \\
\hline & & & & & & & & $\begin{array}{l}\text { Risk } \\
\text { percentile }\end{array}$ & $\begin{array}{l}\text { Prec } \\
1-y\end{array}$ & & $\begin{array}{l}\text { Observed, } \\
1-\mathrm{y}(\%)\end{array}$ \\
\hline & & & & & & & & $\geq 50^{\text {th }}$ & 13.8 & & 13.6 \\
\hline & & & & & & & & $\geq 90^{\text {th }}$ & 36.8 & & 37.7 \\
\hline & & & & & & & & $\geq 98^{\text {th }}$ & 64.6 & & 56.9 \\
\hline & & & & $C$-statistic $\mathrm{N}$ & & & & $\begin{array}{l}C=0.853 \\
C=0.844\end{array}$ & $\begin{array}{l}50-0 . \\
41-0 .\end{array}$ & $\begin{array}{l}\text { 6), wo } \\
\text { 7), me }\end{array}$ & \\
\hline $\begin{array}{l}\text { Crooks et al. } \\
(2016)^{23}\end{array}$ & $\begin{array}{l}1-\mathrm{y} \text { in primary } \\
\text { care patients } \\
\text { age } 20-100 \mathrm{y}\end{array}$ & $\begin{array}{l}\mathrm{n}=328,628 \\
\text { Mean age NR } \\
\% \text { gender NR }\end{array}$ & $\begin{array}{l}\mathrm{n}=328,636 \\
\text { Mean age NR } \\
\% \text { gender NR }\end{array}$ & $\begin{array}{l}\text { NR; used Al } \\
\text { compare rel }\end{array}$ & $\begin{array}{l}\text { ke's } \\
\text { ve go }\end{array}$ & $\begin{array}{l}\text { forma } \\
\text { Iness }\end{array}$ & $\begin{array}{l}\text { on criterion (A } \\
\text { fit }\end{array}$ & ) and likelih & ratic & est (p & $0.0001)$ to \\
\hline & & $3 \% 1-y$ mortality & $3 \% 1-y$ mortality & $C=0.88$ & & & & $C=0.88$ & & & \\
\hline $\begin{array}{l}\text { Pilotto et al. } \\
(2013)^{24}\end{array}$ & $\begin{array}{l}\text { 1-y in } \\
\text { community- } \\
\text { dwelling age }\end{array}$ & $\begin{array}{l}\mathrm{n}=7,876 \\
\text { Mean age } 82 \text { y } \\
63 \% \text { female }\end{array}$ & $\begin{array}{l}\mathrm{n}=4,144 \\
\text { Mean age } 82 \mathrm{y} \\
63 \% \text { female }\end{array}$ & $\begin{array}{l}\text { Quintile of } \\
\text { predicted } \\
\text { risk }\end{array}$ & $\begin{array}{l}\text { Pred } \\
1-y\end{array}$ & ted, & \begin{tabular}{|l} 
Observed, \\
$1-\mathrm{y}(\%)$
\end{tabular} & $\begin{array}{l}\text { Quintile of } \\
\text { predicted } \\
\text { risk }\end{array}$ & $\begin{array}{l}\text { Prec } \\
1-y\end{array}$ & ted, & $\begin{array}{l}\text { Observed, } \\
1-\mathrm{y}(\%)\end{array}$ \\
\hline & $\geq 65 \mathrm{y}$ & $43 \% 1-y$ mortality & $44 \% 1-y$ mortality & 1 & 14.6 & & 11.9 & 1 & 14.6 & & 9.6 \\
\hline & & & & 2 & 25.0 & & 21.2 & 2 & 25.2 & & 23.0 \\
\hline & & & & 3 & 37.7 & & 36.4 & 3 & 38.4 & & 40.4 \\
\hline & & & & 4 & 58.2 & & 66.4 & 4 & 59.4 & & 64.4 \\
\hline & & & & 5 & 91.9 & & 90.2 & 5 & 92.8 & & 91.5 \\
\hline & & & & $C=0.79(0$. & -0.80 & & & $C=0.79(0$. & -0.80 & & \\
\hline $\begin{array}{l}\text { Wang et al. } \\
(2013)^{25}\end{array}$ & $\begin{array}{l}\text { 1-y in primary } \\
\text { care patients } \\
\text { within the } \\
\text { Veterans } \\
\text { Health } \\
\text { Administration }\end{array}$ & $\begin{array}{l}\mathrm{n}=2,761,392 \\
\text { Mean age } 64 \mathrm{y} \\
94 \% \text { male } \\
2.6 \% 1 \text {-y mortality }\end{array}$ & $\begin{array}{l}\mathrm{n}=1,837,016 \\
\text { Random splitting; } \\
\text { can assume similar } \\
\text { characteristics to } \\
\text { development cohort }\end{array}$ & NR & & & & $\begin{array}{l}\text { See Figure } \\
\text { Used Cox c } \\
\text { indicated m } \\
\text { calibrated; } \\
\text { slope }(95 \% \\
\text { intercept } 9\end{array}$ & $\begin{array}{l}\text { (low } \\
\text { oratio } \\
\text { el wa } \\
1.0 \\
\text { CI): }\end{array}$ & $\begin{array}{l}\text { middl } \\
\text { egres } \\
\text { xtrem } \\
(0.99 \\
001(-\end{array}$ & $\begin{array}{l}\text { anel) } \\
\text { n, which } \\
\text { y well- } \\
-1.009) \text {, } \\
21-0.023)\end{array}$ \\
\hline & age $18-110 \mathrm{y}$ & & & C-statistic N & & & & $C=0.851$ & $50-0$. & & \\
\hline Gagne et al. & $1-y$ in & $\mathrm{n}=120,679$ & $\mathrm{n}=123,855$ & NR & & & & Predicted, 1 & $(\%)$ & Obs & ed, 1-y (\%) \\
\hline$(2011)^{26}$ & Medicare & Mean age $80 \mathrm{y}$ & Mean age $79 \mathrm{y}$ & & & & & $<7$ & & 3.1 & \\
\hline & enrollees age & $83 \%$ female & $77 \%$ female & & & & & $7-<17$ & & 11.8 & \\
\hline & $\geq 65 \mathrm{y}$ & $9 \% 1-y$ mortality & $7 \% 1-y$ mortality & & & & & $\geq 17$ & & 29.2 & \\
\hline & & & & $C$-statistic N & & & & $C=0.788$ & $86-0$. & & \\
\hline 15-month all-c & use mortality & & & & & & & & & & \\
\hline $\begin{array}{l}\text { Mazzaglia et } \\
\text { al. }(2007)^{27}\end{array}$ & $\begin{array}{l}15 \text {-mo in } \\
\text { community- }\end{array}$ & $\begin{array}{l}\mathrm{n}=2,470 \\
\text { Mean age } 75 \mathrm{y}\end{array}$ & $\begin{array}{l}\mathrm{N}=2,926 \\
\text { Mean age } 75 \mathrm{y}\end{array}$ & Risk Score & & $\begin{array}{l}\text { Obs } \\
(\%)\end{array}$ & $\begin{array}{l}\text { ved, } 15-\mathrm{mo} \\
5 \% \mathrm{CI})\end{array}$ & Risk Score & & $\begin{array}{l}\text { Obs } \\
(\%)\end{array}$ & $\begin{array}{l}\text { ed, } 15 \text {-mo } \\
5 \% \mathrm{CI})\end{array}$ \\
\hline & dwelling age & $56 \%$ female & $59 \%$ female & 0 & & 0.2 & $04-1.1)$ & 0 & & 0.3( & $3-1.1)$ \\
\hline & $\geq 65 \mathrm{y}$ & $5 \% 15$-mo mortality & $4 \% 15$-mo mortality & 1 & & 1.4 & $4-3.6)$ & 1 & & 0.9 & $1-2.1)$ \\
\hline & & & & 2 & & 1.1 & $4-2.3)$ & 2 & & 0.71 & $2-1.1)$ \\
\hline & & & & $\geq 3$ & & 9.6 & $9-11.5)$ & $\geq 3$ & & 8.2 & 7-9.8) \\
\hline & & & & $C=0.75(0$. & -0.78 & & & $C=0.75(0$. & -0.78 & & \\
\hline 2-year all-caus & nortality & & & & & & & & & & \\
\hline Carey et al. & $2-y$ in & $\mathrm{n}=4,516$ & $\mathrm{n}=2,877$ & Risk Score & & Obs & ved, 3-y (\%) & Risk Score & & Obs & ed, 3-y (\%) \\
\hline$(2004)^{28}$ & community- & Mean age 78 y & Mean age $78 \mathrm{y}$ & $0-2$ & & 3 & & $0-2$ & & 5 & \\
\hline & dwelling age & $61 \%$ female & $61 \%$ female & $3-6$ & & 11 & & $3-6$ & & 12 & \\
\hline & $\geq 70 \mathrm{y}$ & $84 \%$ White & $73 \%$ White & $7-10$ & & 34 & & $7-10$ & & 36 & \\
\hline & & & & $C=0.76$ & & & & $C=0.74$ & & & \\
\hline 3-year all-caus & mortality & & & & & & & & & & \\
\hline $\begin{array}{l}\text { Turusheva et } \\
\text { al. }(2017)^{29}\end{array}$ & \begin{tabular}{|l} 
3-y in \\
community- \\
dwelling adults
\end{tabular} & $\begin{array}{l}\mathrm{n}=379 \\
\text { Mean age } 77 \mathrm{y} \\
75 \% \text { female }\end{array}$ & $\begin{array}{l}\mathrm{n}=567 \\
\text { Mean age } 85 y \\
63 \% \text { female }\end{array}$ & NR & & & & $\begin{array}{l}\text { NR (externa } \\
\text { they show a } \\
\text { of } 0.0011 \text { (9 }\end{array}$ & $\begin{array}{l}\text { Jalida } \\
\text { et recl } \\
\% \text { CI }\end{array}$ & $\begin{array}{l}\mathrm{n} \text { in a } \\
\text { sificat } \\
1742\end{array}$ & $\begin{array}{l}\text { Its } \geq 80) \\
\text { index (NRI) } \\
1884)\end{array}$ \\
\hline & age $\geq 65 y$ & $13 \% 2.5$-y mortality & $23 \% 3-y$ mortality & $\begin{array}{l}C=0.72(0 . \\
C=0.73(0 .\end{array}$ & $\begin{array}{l}-0.74 \\
-0.75 \\
\end{array}$ & $\begin{array}{l}\text { mode } \\
\text { mode }\end{array}$ & & $\begin{array}{l}C=0.59(0 . \\
C=0.60(0 .\end{array}$ & -0.64 & $\begin{array}{l}\text { nodel } \\
\text { model }\end{array}$ & \\
\hline
\end{tabular}


Table 2. Continued

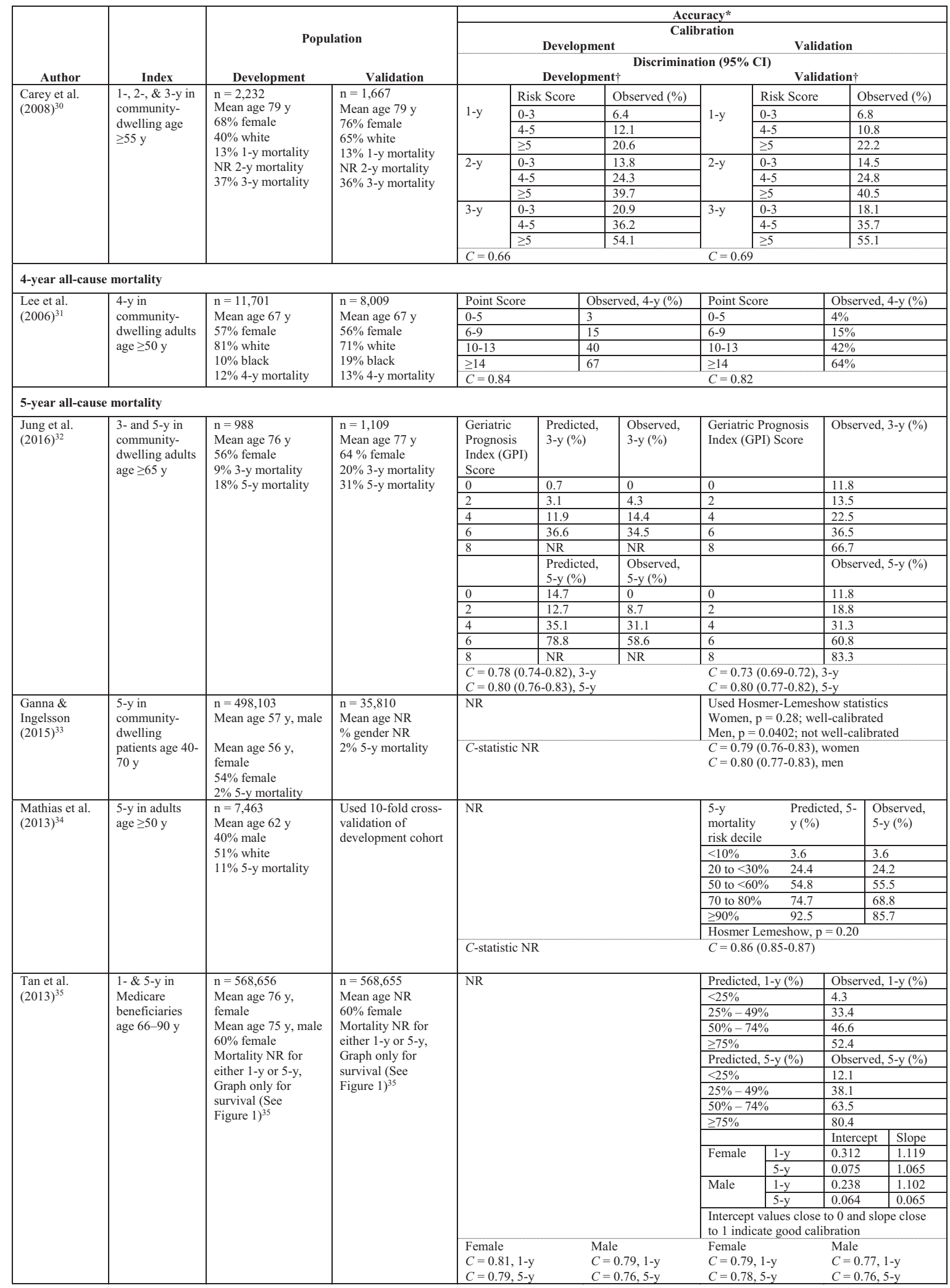


Table 2. Continued

\begin{tabular}{|c|c|c|c|c|c|c|c|c|}
\hline \multirow[b]{5}{*}{ Author } & \multirow[b]{5}{*}{ Index } & \multirow{3}{*}{\multicolumn{2}{|c|}{ Population }} & \multicolumn{5}{|c|}{ Accuracy* } \\
\hline & & & & \multicolumn{5}{|c|}{ Calibration } \\
\hline & & & & \multicolumn{4}{|c|}{ Development } & Validation \\
\hline & & \multirow[b]{2}{*}{ Development } & \multirow[b]{2}{*}{ Validation } & \multicolumn{5}{|c|}{ Discrimination $(95 \% \mathrm{CI})$} \\
\hline & & & & \multicolumn{3}{|c|}{ Development $\dagger$} & \multicolumn{2}{|c|}{ Validation $\dagger$} \\
\hline \multirow[t]{14}{*}{$\begin{array}{l}\text { Zhang et al. } \\
(2012)^{36}\end{array}$} & \multirow{14}{*}{$\begin{array}{l}1-\& 5 \text {-y in } \\
\text { community- } \\
\text { dwelling adults } \\
\text { age } \geq 70 \mathrm{y}\end{array}$} & \multirow{14}{*}{$\begin{array}{l}\mathrm{n}=4,434 \\
49.7 \% \text { age } \geq 70-<75 \mathrm{y} \\
63.3 \% \text { female } \\
89.2 \% \text { white } \\
3.3 \% 1 \text {-y mortality } \\
22.8 \% 5 \text {-y mortality }\end{array}$} & \multirow{14}{*}{$\begin{array}{l}\mathrm{n}=2,939 \\
49.3 \% \text { age } \geq 70-<75 \mathrm{y} \\
62.4 \% \text { female } \\
88.3 \% \text { white } \\
4.1 \% 1-\mathrm{y} \text { mortality } \\
24.0 \% 5 \text {-y mortality }\end{array}$} & \multicolumn{2}{|c|}{$\begin{array}{l}\text { Risk Groups (Sum } \\
\text { Scores) }\end{array}$} & Observed, 1-y (\%) & $\begin{array}{l}\text { Risk Groups } \\
\text { (Sum Scores) }\end{array}$ & Observed, 1-y (\%) \\
\hline & & & & \multicolumn{2}{|c|}{ Very Low $(0-2)$} & 1.8 & Very Low $(0-2)$ & 2.0 \\
\hline & & & & \multicolumn{2}{|l|}{ Low (3-4) } & 4.7 & Low (3-4) & 6.5 \\
\hline & & & & \multicolumn{2}{|l|}{ Moderate (5-6) } & 7.6 & Moderate (5-6) & 11.5 \\
\hline & & & & \multicolumn{2}{|l|}{ High (7-8) } & 21.9 & High $(7-8)$ & 18.8 \\
\hline & & & & \multirow{2}{*}{\multicolumn{2}{|c|}{ Very High $(\geq 9)$}} & 47.1 & Very High $(\geq 9)$ & 31.6 \\
\hline & & & & & & Observed, 5-y (\%) & & Observed, 5-y (\%) \\
\hline & & & & \multicolumn{2}{|c|}{ Very Low $(0-2)$} & 6.9 & Very Low $(0-2)$ & 8.7 \\
\hline & & & & \multicolumn{2}{|l|}{ Low (3-6) } & 12.8 & Low (3-6) & 14.5 \\
\hline & & & & \multicolumn{2}{|c|}{$\begin{array}{l}\text { Moderate (7-14) } \\
\text { High }(15-17)\end{array}$} & 30.0 & Moderate (7-14) & 30.3 \\
\hline & & & & \multicolumn{2}{|c|}{$\begin{array}{l}\text { High }(15-17) \\
\text { Very High }(>18)\end{array}$} & 55.0 & High (15-17) & 57.6 \\
\hline & & & & Very High $(\geq 1$ & & 79.9 & Very High $(\geq 18)$ & 66.1 \\
\hline & & & & \multicolumn{5}{|c|}{$\begin{array}{l}\text { Hosmer-Lemeshow statistics } \mathrm{p}>0.1 \text { for } 1-\mathrm{y} \text { and } 5-\mathrm{y} \text {, which indicates good overall } \\
\text { calibration }\end{array}$} \\
\hline & & & & \multicolumn{3}{|c|}{$\begin{array}{l}C=0.72,1-\mathrm{y} \\
C=0.74,5-\mathrm{y}\end{array}$} & \multicolumn{2}{|l|}{$\begin{array}{l}C=0.72,1-\mathrm{y} \\
C=0.72,5-\mathrm{y}\end{array}$} \\
\hline \multirow[t]{7}{*}{$\begin{array}{l}\text { Schonberg et } \\
\text { al. }(2009)^{37}\end{array}$} & \multirow{7}{*}{$\begin{array}{l}5 \text {-y in } \\
\text { community- } \\
\text { dwelling adults } \\
\text { age }>65 \mathrm{y}\end{array}$} & \multirow{7}{*}{$\begin{array}{l}\mathrm{n}=16,077 \\
27 \% \text { age } \geq 80 \mathrm{y} \\
62 \% \text { female } \\
85 \% \text { non-Hispanic } \\
\text { white } \\
17 \% 5 \text {-y mortality }\end{array}$} & $\begin{array}{l}\mathrm{n}=8,038 \\
\text { Random splitting; }\end{array}$ & Point Score & $\begin{array}{l}\mathrm{Ob} \\
(95\end{array}$ & $\begin{array}{l}\text { red, } 5-y(\%) \\
\text { CI) }\end{array}$ & Point Score & $\begin{array}{l}\text { Observed, } 5-y(\%) \\
(95 \% \mathrm{CI})\end{array}$ \\
\hline & & & can assume similar & $0-1$ & $2(1$ & & $0-1$ & $3(1-6)$ \\
\hline & & & characteristics to & $6-7$ & 11 & -14) & $6-7$ & $12(10-15)$ \\
\hline & & & development cohort & $10-11$ & 25 & $-28)$ & $10-11$ & $29(25-33)$ \\
\hline & & & & $14-15$ & 47 & $-42)$ & $14-15$ & $49(43-55)$ \\
\hline & & & & $\geq 18$ & 71 & -77) & $\geq 18$ & $62(54-70)$ \\
\hline & & & & $C$-statistic NR & & & $C=0.75$ & \\
\hline
\end{tabular}

NA, not available; NR, not reported; CI, confidence interval.

Data for 6-month to 5 -year mortality only, with $95 \%$ confidence interval if reported.

$\dagger$ Predicted and observed mortality rate or ranges of mortality rate, from low to high risk groups if reported (e.g., percentiles or classes [low, middle, or high]).

well calibrated across all risk groups and had good discrimination.

Wang et al. ${ }^{25}$ developed a model to predict 1 year mortality in patients aged 18 to 100 years who were assigned to a Veterans Health Administration primary care provider. It was well calibrated (Cox Intercept, $\alpha=0.001$ [95\% CI, -0.001 to 0.023 ; Cox Slope, $\beta=1.002$ [95\% CI, 0.998-1.008]) and had very good discrimination. However, a narrow range of mortality was observed $(0.1 \%$ to $9.1 \%)$ for patients categorized between the $5^{\text {th }}$ and $90^{\text {th }}$ predicted risk percentiles. Coefficients included in the model to predict death are available in a supplemental table with $95 \%$ CIs.

Gagne et al. ${ }^{26}$ used low-income Medicare enrollees from Pennsylvania and New Jersey. The index was well calibrated and had good discrimination. Mortality ranged from 3\% to $29 \%$.

\section{Fifteen-Month Mortality}

Mazzaglia et al. ${ }^{27}$ developed and validated a $15-$ month mortality index for community-dwelling older adults by using data from a screening survey of patients answered by primary care physicians from 2 regions of Florence, Italy. The final model includes a number of positive responses to another screening test, which is not available in the article. ${ }^{45}$ The index was well calibrated and showed good discrimination. The reported mortality was narrow, ranging from $0 \%$ to $10 \%$.

\section{Two-Year Mortality}

Carey et al. $^{28}$ developed a functional morbidity index to predict 2-year mortality in communitydwelling older adults aged 70 and older by using data from the Asset and Health Dynamics Among the Oldest Old study in the United States. The index was well calibrated across the risk groups and demonstrated good discrimination.

\section{Three-Year Mortality}

Turusheva et al. ${ }^{29}$ developed 2 models of mortality risk score to predict 3-year mortality. The derivation cohort $(\mathrm{n}=379)$ was randomly sampled using data from a prospective cohort study of community-dwelling older adults aged 65 to 91 years in Saint Petersburg, Russia. The authors validated the 2 models by using a cohort from an external cohort study of people aged 80 years or older in Belgium $(\mathrm{n}=567)$. Both models had good discrimination in the development cohort but poor-to-moderate performance in validation. Calibration was not reported in the study.

The other 3-year mortality index for communitydwelling elderly was developed by Carey et al. ${ }^{30}$ This index allows for prediction of 1-, 2-, and 3-year 


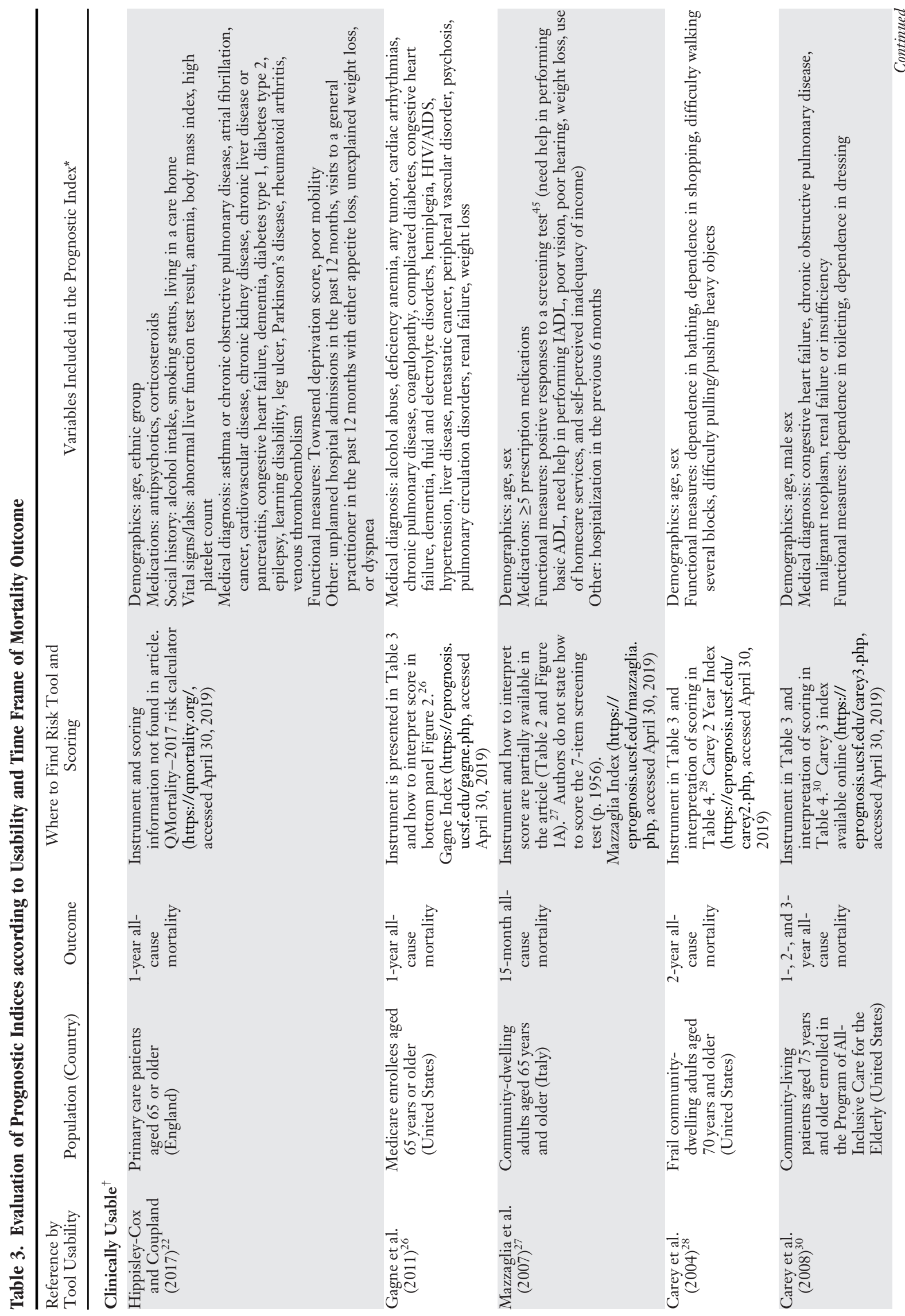




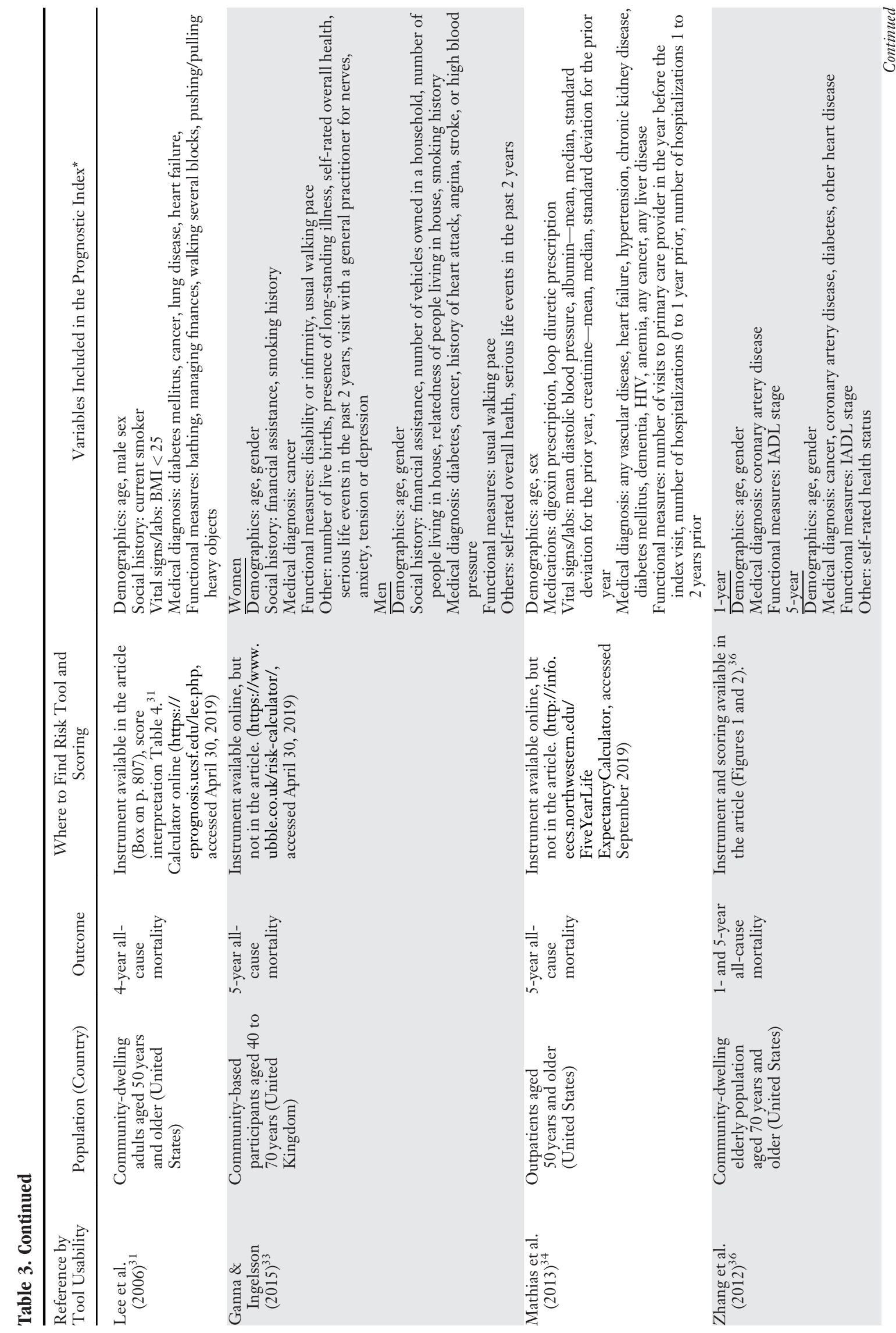




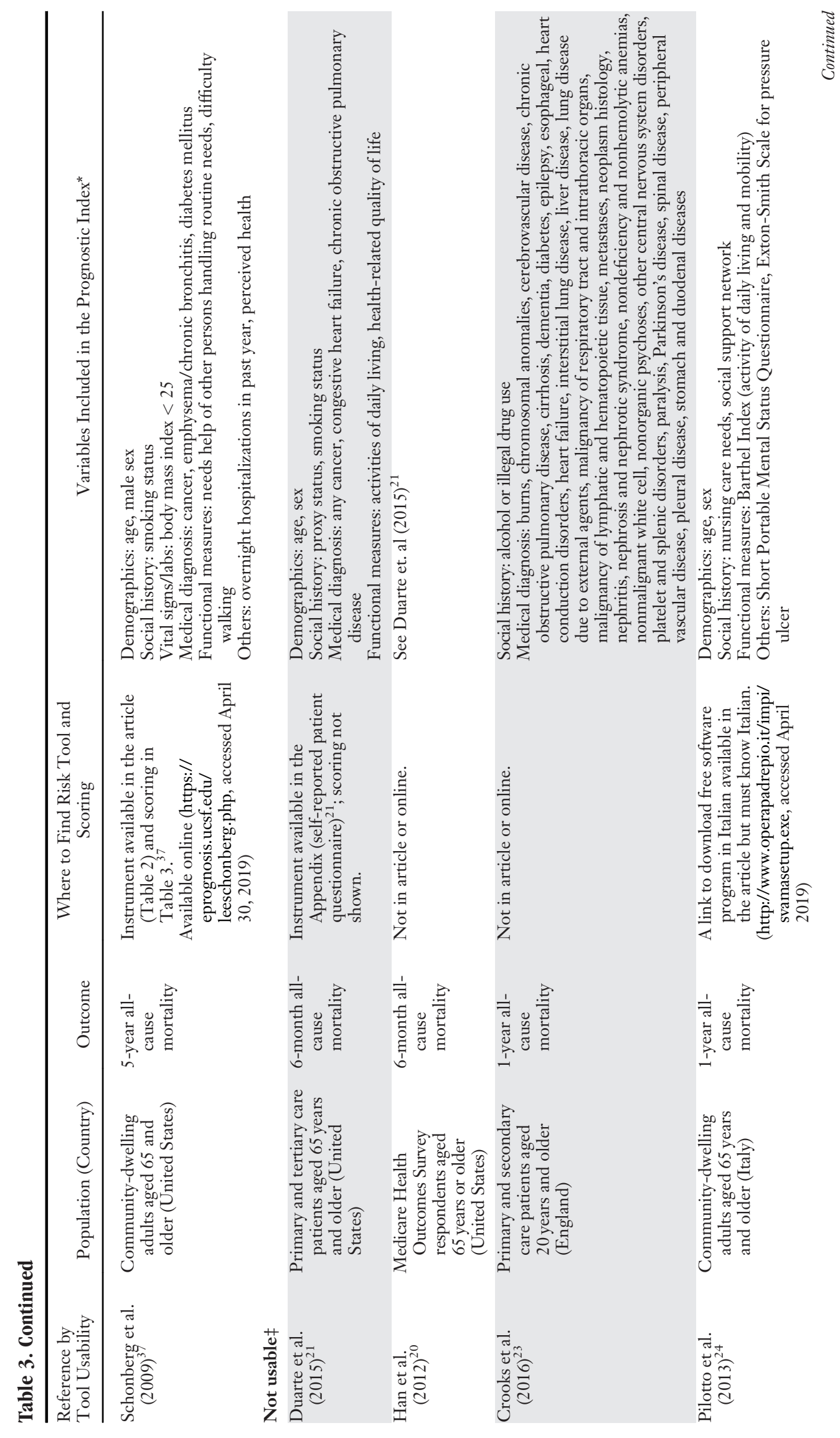




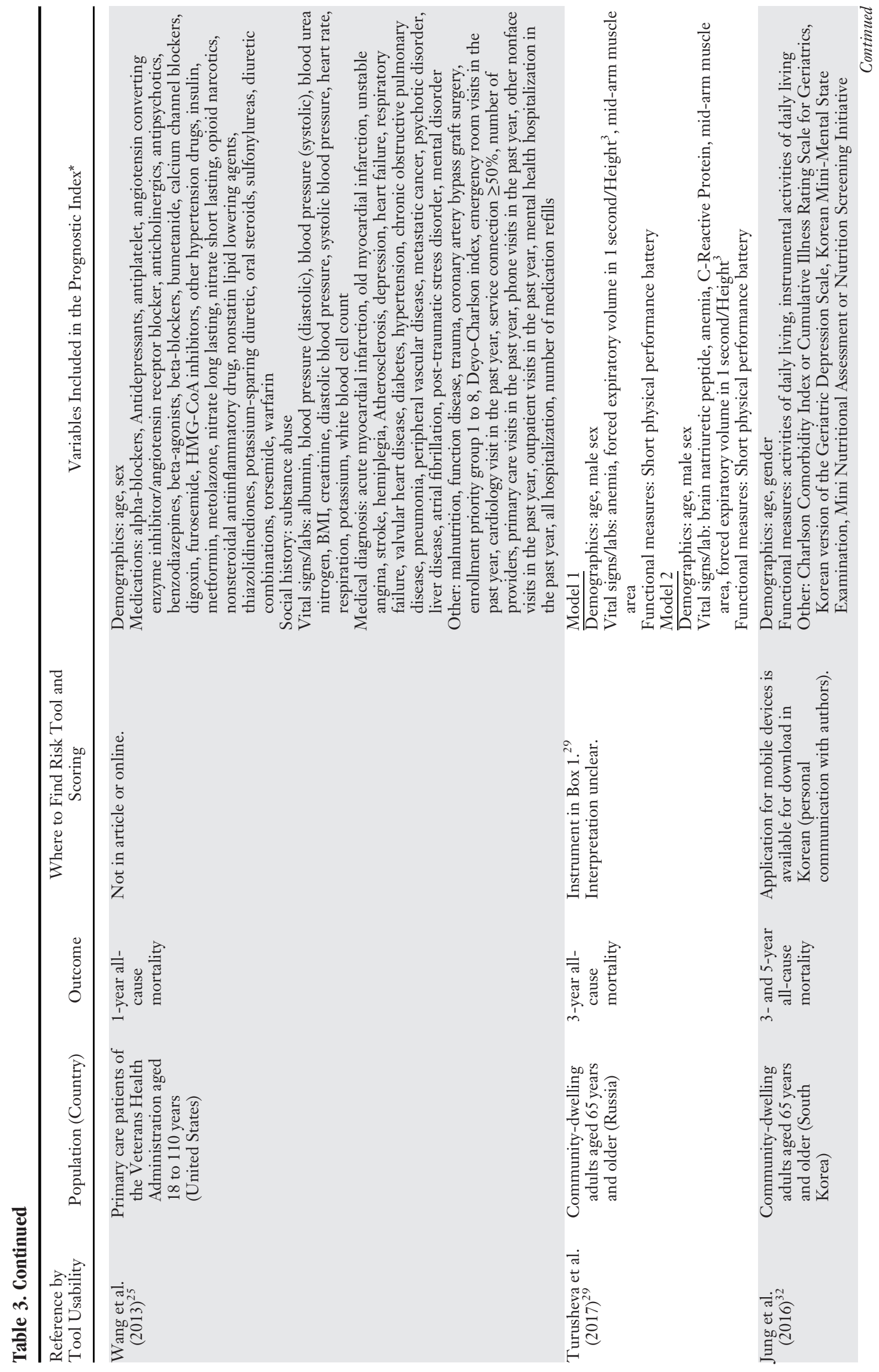




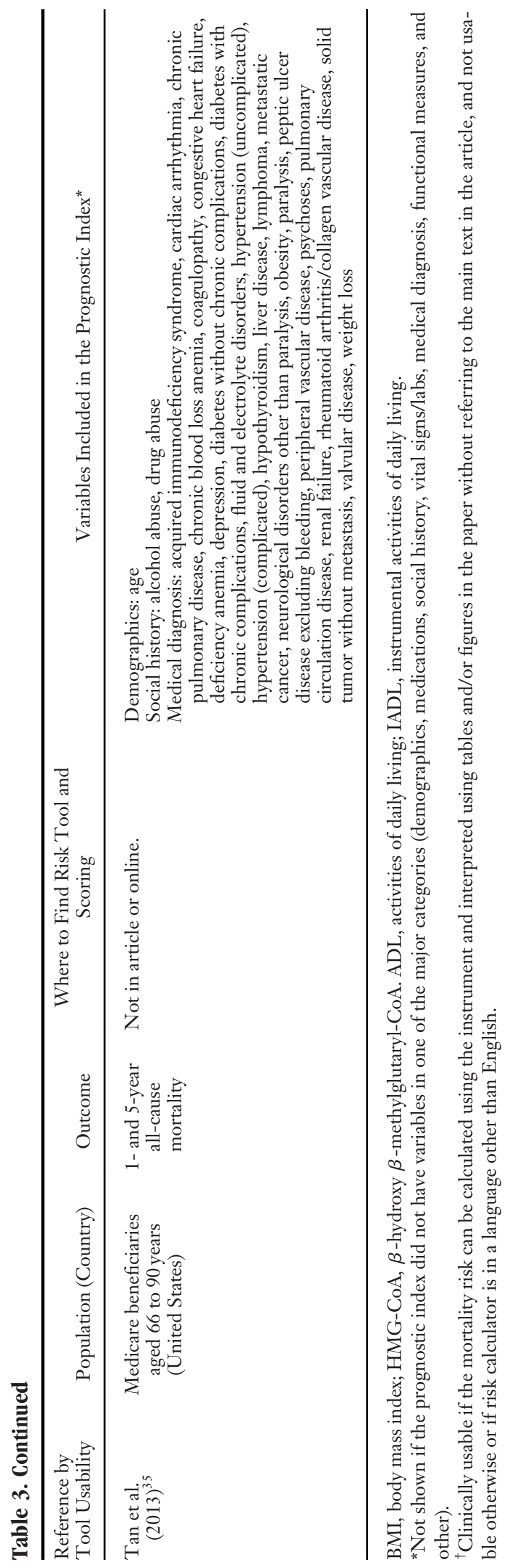

mortality. Its data source was patients enrolled in the Program of All-inclusive Care for the Elderly in the Western, Midwestern, and Eastern regions of the United States. The index was developed using the cohort from the Western region and validated in the other 2 regions. The index had moderate discrimination and was well calibrated across all risk groups for 1-, 2-, and 3-year mortality.

\section{Four-Year Mortality}

A 4-year mortality index was developed by Lee et al. $^{31}$ in community-dwelling adults aged 50 years and older in the United States who answered the Health and Retirement Survey from 1992 to 1998. The development and validation cohorts were chosen based on geographic location in the United States. The index was well calibrated across all risk groups and had very good discrimination.

\section{Five-Year Mortality}

Jung et al. ${ }^{32}$ developed a geriatric prognosis index to predict 3- and 5-year mortality. Its data source for development was the Korean Longitudinal Study on Health and Aging cohort, which included people aged 65 years and older living in a suburban city of South Korea. A retrospective review of medical records of people aged 60 years and older who had a geriatric assessment in the outpatient geriatric clinic or inpatient ward was used for validation. The proportion of inpatients used for the validation cohort was not reported. The index requires the use of a number of other scores such as the Charlson Comorbidity Index and multiple geriatric scales. Three-year mortality was well calibrated for all risk groups. Calibration for 5-year mortality was poor for higher risk groups but well calibrated for lower and middle risk groups. For both 3- and 5year mortality, the $95 \%$ CI for mortality was wide for all risk groups. The index had good discrimination for 3-year mortality and very good discrimination for 5-year mortality.

Ganna and Ingelsson ${ }^{33}$ developed a 5-year mortality prediction score using UK Biobank participant data from England and Wales, and they validated it using participants from Scotland. Prediction models were developed separately for men (13 items) and women (11 items). These models had very good discrimination for men and good discrimination for women. The score for men was poorly calibrated (Hosmer-Lemeshow, $P=.0402$ ), 
Figure 2. Flow diagram of study selection process to identify potentially useful prognostic indices in the primary care setting to help initiate advance care planning, adapted from the PRISMA statement. ${ }^{24}$ Abbreviations: MeSH, Medical Subject Headings; ICU, intensive care unit

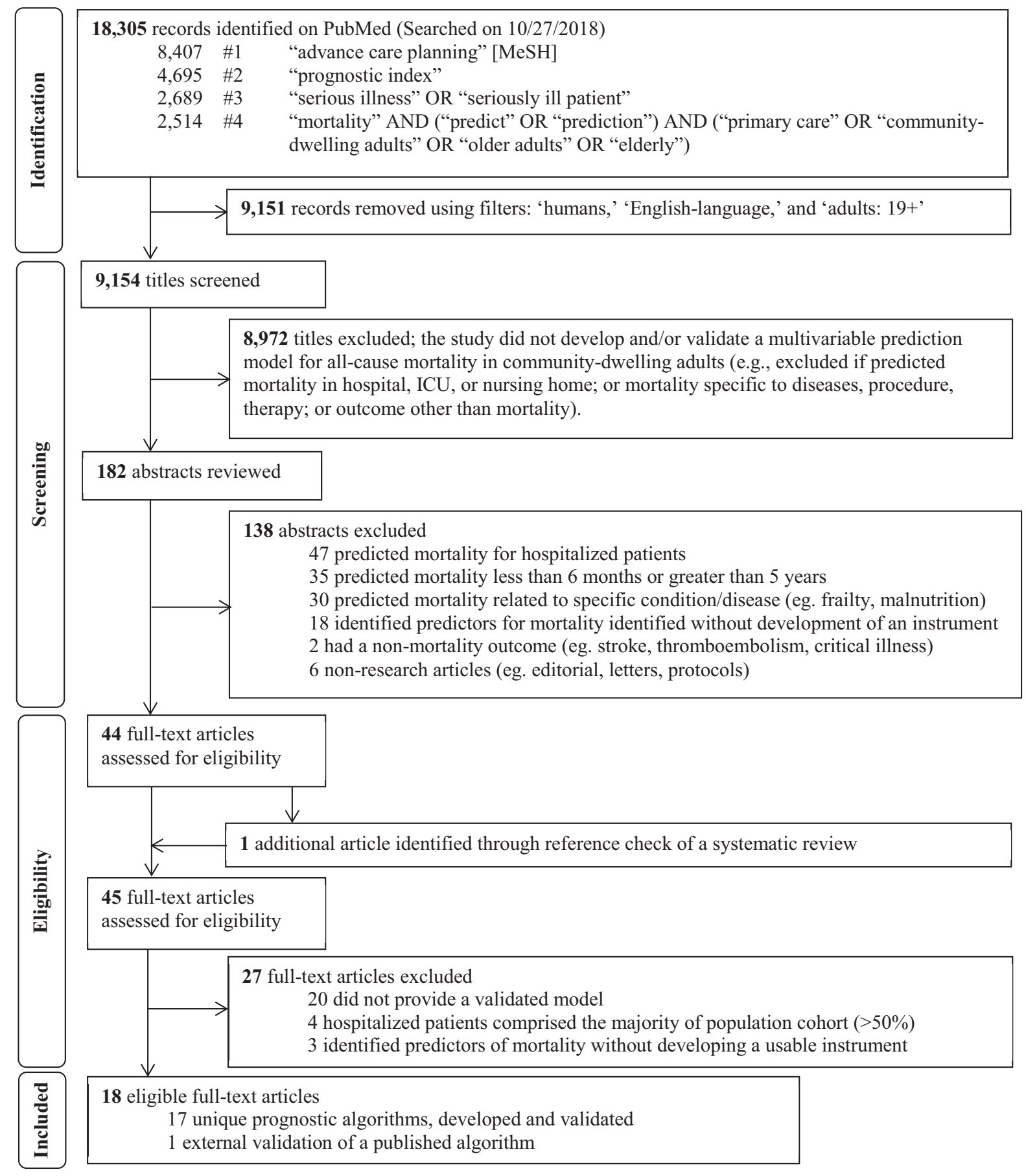

but the score for women was well calibrated (Hosmer-Lemeshow, $P=.28$ ). For the HosmerLemeshow test, statistical significance $(P<.05)$ means poor calibration.

The Ensemble Index developed by Mathias et al. ${ }^{34}$ to predict 5 -year mortality was developed using predictive data mining and analysis of electronic health records data from Epic (Verona, WI) and Cerner (Kansas City, MO). The random forest ensemble technique with alternating decision tree was used to develop the model, and 10-fold cross validation was used. Its discrimination was very good, 
showing a higher c-statistic than the Walter life expectancy method and Charlson Comorbidity Index, ${ }^{46,47}$ and it was well calibrated across all risk groups.

Tan et al. ${ }^{35}$ developed a life expectancy model that adapts the Elixhauser comorbidity measure ${ }^{44}$ to predict 1 - and 5-year mortality in the Medicare population in the United States. A 5\% random sample of Medicare data was randomly split for development and validation. The model was well calibrated for all risk groups for 5-year mortality and for low-to-middle risk groups for 1-year mortality in both males and females. Calibration in the high-risk groups for 1-year mortality in both males and females was poor. Discrimination was very good for 1-year mortality in females and good for 5-year mortality in females and 1- and 5year mortality in males.

Zhang et al. ${ }^{36}$ developed a 1 - and 5 -year mortality index using data collected alongside a national health survey of noninstitutionalized adults in the United States. The development cohort came from randomly selecting $60 \%$ and using the remaining $40 \%$ for validation. The models were well calibrated according to the Hosmer-Lemeshow statistics. Both the 1- and 5-year mortality index had good discrimination and predicted a wide range of mortality between low- and high-risk groups (2\% to $42 \%$ for 1 year and $7 \%$ to $81 \%$ for 5 year).

Schonberg et al. ${ }^{37}$ developed a 5 -year mortality index for adults aged 65 years and older with good discrimination. Linking data from the National Health Interview Survey and the National Death Index, two-thirds were randomly selected for development and the remainder for validation. The index was well calibrated across all risk groups and predicted a wide range of mortality between the lowest to highest risk groups.

\section{Discussion}

This review summarizes 17 unique prognostic indices from 18 articles that predict all-cause mortality between 6 months to 5 years in community-dwelling adults. Our review summarizes the performance of prognostic indices and assesses their potential for clinical use aimed at supporting implementation of ACP in the primary care setting. Ten articles included algorithms that were usable in the setting of primary care office. ${ }^{22,26-28,30,31,33,34,36,37}$ Our search criteria included adults 18 years and older. However, only 3 of the 10 usable indices were developed and validated in a population cohort that included patients less than 65 years. ${ }^{31,33,34}$ Three systematic reviews have identified prognostic indices that predict mortality in communitydwelling adults, ${ }^{38,48,49}$ but none of them made recommendations on which tool to prioritize for clinical implementation. Even a prognostic index that is accurate, externally validated, well calibrated, and with a low risk of bias may still have limited clinical use and impact if it is difficult to use and if the physician does not have access to all variables necessary for a specific prognostic algorithm. Several of the indices we identified as clinically usable require knowledge of multiple variables and are impractical without systematic collection of these variables or additional programming in an electronic medical record.

\section{Implications for Future Research}

Currently available approaches to prognostication include clinical intuition and algorithms. A validated approach using clinical intuition to trigger palliative care is to ask the following Surprise Question (SQ): "Would I be surprised if this patient died in the next 12 months?"17 Because the SQ was not originally developed to predict mortality, more research is needed to test how the SQ can aid in the patient identification process for physicians to initiate ACP. ${ }^{16}$ Combining the SQ with another prognostic tool has the potential to enhance accuracy in determining a patient's prognosis. $^{50}$

The indices we classified as clinically usable may not be easy to use. They often require knowledge of many variables that may not be easily accessible to the practicing physician. Future research should compare the clinically usable indices we identified for time spent per patient and resources required to program them into their existing electronic medical records to see which ones are most feasible in busy practices, given the large number of variables that many of them have..$^{22,26-28,30,31,33,34,36,37}$ It is possible that these algorithms could be programmed into the electronic medical record to prompt physicians to discuss ACP with appropriate patients, the same way many other best practice alerts are now. It is currently unclear which if any of the indices we identified might work best for initiating ACP discussions. With a growing interest in the use of 
machine learning and artificial intelligence in medical care, our results can guide researchers who wish to test multiple algorithms simultaneously. ${ }^{51,52}$

Our work has implications for practice-based research networks that wish to expand the implementation of ACP in the primary care setting. For example, the Patient-Centered Outcomes Research Institute recently funded 7 studies to encourage the expansion of ACP and palliative care. ${ }^{53,54}$ The Meta-network Learning And Research Center (Meta-LARC) ACP trial is 1 of these studies. ${ }^{55}$ Meta-LARC is a consortium of 7 practice-based research networks in the United States and Canada including over 900 primary care practices and approximately 4000 clinicians who care for over 3 million patients. Meta-LARC is dedicated to increasing the quality, effectiveness, and safety of primary care through accelerated research and collaborative learning (https://www.ohsu.edu/ oregon-rural-practice-based-research-network/ meta-larc). The ACP trial will use the infrastructure of Meta-LARC to conduct a cluster randomized trial in 42 primarily family physician practices in the United States and Canada to compare the efficacy of clinician-led versus team-based approaches to implement ACP in primary care.

\section{Limitations}

The risk of bias in individual indices was not assessed, as it was not applicable for our review. Publication bias may exist because we searched only on PubMed, which may miss some articles. Given the heterogeneity in the way studies reported their calibration, straightforward comparisons were impossible. Studies that included administrative data may have included hospitalized and nursing home patients. Although we attempted to exclude indices developed on cohorts where more than $50 \%$ were hospitalized or in nursing homes, not all articles provided this information. For this study, we abstracted the calibration statistics, as reported by the authors of each prognostic index. Currently, methods to assess model performance are not standardized and are reported in a variety of ways. Future studies of prognostic indices should report calibration by using standard means. ${ }^{42}$ Clinicians and researchers can choose to implement the prognostic algorithms we classified as usable and test whether appropriate patients for ACP conversations are identified in the primary care setting.

\section{Conclusion}

Our review identified 18 studies with 17 published prognostic indices that are potentially useful for patient identification for ACP conversations. Eight prognostic indices from the United States and 2 from the United Kingdom were identified as clinically usable. $^{22,26-28,30,31,33,34,36,37}$ An index classified as clinically usable may not be easy to use because of a large number of variables that are not routinely collected and the need for programming the index into the electronic medical record. Future research should validate these indices in other populations, compare across indices to determine time spent per patient, and program them into electronic medical records to see which ones are most feasible in busy practices.

The authors thank Sharon Straus, MD, University of Toronto, Toronto, Ontario, and Annette Totten, $\mathrm{PhD}$, Oregon Health and Science University, Portland, Oregon for their feedback on this manuscript. The authors would like to thank Parang Kim, MS, University of Iowa, for her work in formatting the tables.

To see this article online, please go to: http://jabfm.org/content/ 33/2/322.full.

\section{References}

1. Jimenez G, Tan WS, Virk AK, Low CK, Car J, Ho A. Overview of systematic reviews of advance care planning: summary of evidence and global lessons. J Pain Symptom Manage 2018;56:436-59.

2. United States Government Accountability Office. Advance care planning: selected states' efforts to educate and address access challenges. 2019. https:// www.gao.gov/assets/700/696985.pdf. Accessed March 6, 2019.

3. Rietjens JAC, Sudore RL, Connolly $M$, et al. Definition and recommendations for advance care planning: an international consensus supported by the European Association for Palliative Care. Lancet Oncol 2017;18:e543-e551.

4. Sudore RL, Lum HD, You JJ, et al. Defining advance care planning for adults: a consensus definition from a multidisciplinary Delphi panel. J Pain Symptom Manage 2017;53:821-32.e821.

5. Brinkman-Stoppelenburg A, Rietjens JA, van der Heide A. The effects of advance care planning on end-of-life care: a systematic review. Palliat Med 2014;28:1000-25.

6. Houben CHM, Spruit MA, Groenen MTJ, Wouters EFM, Janssen DJA. Efficacy of advance care planning: a systematic review and meta-analysis. J Am Med Dir Assoc 2014;15:477-89.

7. Detering KM, Hancock AD, Reade MC, Silvester $W$. The impact of advance care planning on end of 
life care in elderly patients: randomised controlled trial. BMJ 2010;340:c1345.

8. Bischoff KE, Sudore R, Miao Y, Boscardin WJ, Smith AK. Advance care planning and the quality of end-of-life care in older adults. J Am Geriatr Soc 2013;61:209-14.

9. Yadav KN, Gabler NB, Cooney E, et al. Approximately one in three US adults completes any type of advance directive for end-of-life care. Health Aff (Millwood) 2017;36:1244-51.

10. Lakin JR, Koritsanszky LA, Cunningham R, et al. A systematic intervention to improve serious illness communication in primary care. Health Aff (Millwood) 2017;36:1258-64.

11. Sudore RL, Heyland DK, Lum HD, et al. Outcomes that define successful advance care planning: a Delphi panel consensus. J Pain Symptom Manage 2018;55:245-55.e8.

12. Committee on Approaching Death: Addressing Key End of Life Issues; Institute of Medicine. Dying in America: improving quality and honoring individual preferences near the end of life. Washington, DC: National Academies Press (US); 2015.

13. De Vleminck A, Houttekier D, Pardon K, et al. Barriers and facilitators for general practitioners to engage in advance care planning: a systematic review. Scand J Pri Health Care 2013;31:215-26.

14. Stuart B. The NHO Medical Guidelines for NonCancer Disease and local medical review policy: hospice access for patients with diseases other than cancer. Hosp J 1999;14:139-54.

15. Thomas K, Noble B. Improving the delivery of palliative care in general practice: an evaluation of the first phase of the Gold Standards Framework. Palliat Med 2007;21:49-53.

16. Thomas K. Improving end of life care: a matter of life and death. London J Prim Care (Abingdon) 2009;2:89-92.

17. Billings JA, Bernacki R. Strategic targeting of advance care planning interventions: the Goldilocks phenomenon. JAMA Intern Med 2014;174:620-4.

18. Tricco AC, Lillie E, Zarin W, et al. PRISMA Extension for scoping reviews (PRISMA-ScR): checklist and explanation. Ann Intern Med 2018;169:467-73.

19. Moons KG, Altman DG, Reitsma JB, et al. Transparent reporting of a multivariable prediction model for individual prognosis or diagnosis (TRIPOD): explanation and elaboration. Ann Intern Med 2015;162:W1-73.

20. Han PK, Lee M, Reeve BB, et al. Development of a prognostic model for six-month mortality in older adults with declining health. J Pain Symptom Manage 2012;43:527-39.

21. Duarte CW, Black AW, Murray K, et al. Validation of the patient-reported outcome mortality Prediction tool (PROMPT). J Pain Symptom Manage 2015;50:241-7.e6.
22. Hippisley-Cox J, Coupland C. Development and validation of QMortality risk prediction algorithm to estimate short term risk of death and assess frailty: cohort study. BMJ 2017;358:j4208.

23. Crooks CJ, Card TR, West J. The use of a Bayesian hierarchy to develop and validate a co-morbidity score to predict mortality for linked primary and secondary care data from the NHS in England. PLoS One 2016;11:e0165507.

24. Pilotto A, Gallina P, Fontana A, et al. Development and validation of a Multidimensional Prognostic Index for mortality based on a standardized Multidimensional Assessment Schedule (MPISVaMA) in community-dwelling older subjects. J Am Med Dir Assoc 2013;14:287-92.

25. Wang L, Porter B, Maynard C, et al. Predicting risk of hospitalization or death among patients receiving primary care in the Veterans Health Administration. Med Care 2013;51:368-73.

26. Gagne JJ, Glynn RJ, Avorn J, Levin R, Schneeweiss S. A combined comorbidity score predicted mortality in elderly patients better than existing scores. J Clin Epidemiol 2011;64:749-59.

27. Mazzaglia G, Roti L, Corsini G, et al. Screening of older community-dwelling people at risk for death and hospitalization: the Assistenza Socio-Sanitaria in Italia project. J Am Geriatr Soc 2007;5 5:1955-60.

28. Carey EC, Walter LC, Lindquist K, Covinsky KE. Development and validation of a functional morbidity index to predict mortality in community-dwelling elders. J Gen Intern Med 2004;19:1027-33.

29. Turusheva A, Frolova E, Bert V, Hegendoerfer E, Degryse JM. Validation of a new mortality risk prediction model for people 65 years and older in northwest Russia: the Crystal risk score. Arch Gerontol Geriatr 2017;71:105-14.

30. Carey EC, Covinsky KE, Lui LY, Eng C, Sands LP, Walter LC. Prediction of mortality in community-living frail elderly people with long-term care needs. J Am Geriatr Soc 2008;56:68-75.

31. Lee SJ, Lindquist K, Segal MR, Covinsky KE. Development and validation of a prognostic index for 4-year mortality in older adults. JAMA 2006; 295:801-8.

32. Jung HW, Kim JW, Han JW, et al. Multidimensional Geriatric Prognostic Index, based on a geriatric assessment, for long-term survival in older adults in Korea. PLoS One 2016;11:e0147032.

33. Ganna A, Ingelsson E. 5 year mortality predictors in 498,103 UK Biobank participants: a prospective population-based study. Lancet 2015;386:533-40.

34. Mathias JS, Agrawal A, Feinglass J, Cooper AJ, Baker DW, Choudhary A. Development of a 5 year life expectancy index in older adults using predictive mining of electronic health record data. J Am Med Inform Assoc 2013;20:e118-124.

35. Tan A, Kuo YF, Goodwin JS. Predicting life expectancy for community-dwelling older adults from 
Medicare claims data. Am J Epidemiol 2013;178: 974-83.

36. Zhang Z, Xie D, Kurichi JE, Streim J, Zhang G, Stineman MG. Mortality predictive indexes for the community-dwelling elderly US population. J Gen Intern Med 2012;27:901-10.

37. Schonberg MA, Davis RB, McCarthy EP, Marcantonio ER. Index to predict 5-year mortality of community-dwelling adults aged 65 and older using data from the National Health Interview Survey. J Gen Intern Med 2009;24:1115-22.

38. Yourman LC, Lee SJ, Schonberg MA, Widera EW, Smith AK. Prognostic indices for older adults: a systematic review. JAMA 2012;307:182-92.

39. Hanley JA, McNeil BJ. The meaning and use of the area under a receiver operating characteristic (ROC) curve. Radiology 1982;143:29-36.

40. Steyerberg EW, Vickers AJ, Cook NR, et al. Assessing the performance of prediction models: a framework for traditional and novel measures. Epidemiology 2010;21:128-38.

41. Moher D, Liberati A, Tetzlaff J, Altman DG, The PRISMA Group. Preferred reporting items for systematic reviews and meta-analyses: the PRISMA statement. PLoS Med 2009;6:e1000097.

42. Alba AC, Agoritsas T, Walsh M, et al. Discrimination and calibration of clinical prediction models: users' guides to the medical literature. JAMA 2017;318:1377-84.

43. Charlson ME, Pompei P, Ales KL, MacKenzie CR. A new method of classifying prognostic comorbidity in longitudinal studies: development and validation. J Chronic Dis 1987;40:373-83.

44. Elixhauser A, Steiner C, Harris DR, Coffey RM. Comorbidity measures for use with administrative data. Med Care 1998;36:8-27.

45. Roti L, Corsini G, Colombini A, et al,. L. A screening instrument to identify older community-dwellers at risk for death and hospitalization in Tuscany, Italy. The "Assistenza Socio-Sanitaria in Italia" project. J Am Geriatr Soc 2006;54:S90-S91.

46. Walter LC, Covinsky KE. Cancer screening in elderly patients: a framework for individualized decision making. JAMA 2001;285:2750-6.
47. Deyo RA, Cherkin DC, Ciol MA. Adapting a clinical comorbidity index for use with ICD-9-CM administrative databases. J Clin Epidemiol 1992; 45:613-9.

48. O'Caoimh R, Cornally N, Weathers E, et al. Risk prediction in the community: a systematic review of case-finding instruments that predict adverse healthcare outcomes in community-dwelling older adults. Maturitas 2015;82:3-21.

49. National Institute for Health and Care Excellence. Multimorbidity: assessment, prioritisation and management of care for people with commonly occurring multimorbidity. London: National Institute for Health and Care Excellence (UK); 2016.

50. Lakin JR, Robinson MG, Bernacki RE, et al. Estimating 1-year mortality for high-risk primary care patients using the "surprise" question. JAMA Intern Med 2016;176:1863-5.

51. Rose S. Mortality risk score prediction in an elderly population using machine learning. Am J Epidemiol 2013;177:443-52.

52. Udelsman B, Chien I, Ouchi K, Brizzi K, Tulsky JA, Lindvall C. Needle in a haystack: natural language processing to identify serious illness. J Palliat Med 2019;22:179-82.

53. Patient-Centered Outcomes Research Institute (PCORI). Cycle 32016 funding cycle PCORI funding annoucement: community-based palliative care delivery for adult patients with advanced illnesses and their caregivers. PCORI website. https:// www.pcori.org/sites/default/files/PCORI-PFA2016-Cycle-3-Palliative-Care.pdf. Updated May 19, 2017. Accessed April 10, 2019.

54. U.S. National Library of Medicine. Team-based versus primary care clinician-led advance care planning in practice-based research networks. ClinicalTrials.gov website. https://ClinicalTrials. gov/show/NCT03577002. Updated December 3, 2018. Accessed March 6, 2019.

55. Totten AM, Fagnan LJ, Dorr D, et al. Protocol for a cluster randomized trial comparing teambased to clinician-focused implementation of advance care planning in primary care. J Palliat Med 2019;22:82-9. 